\title{
Primary prevention of acute rheumatic fever
}

CPD

Rosemary Wyber, Asha C Bowen, Anna P Ralph, David Peiris

\section{Background}

Acute rheumatic fever (ARF) is an abnormal immune reaction following Streptococcus pyogenes (Strep A) infection of the throat, and likely the skin. Primary prevention is the prompt and appropriate antibiotic treatment of Strep A infection, and it can reduce the risk of developing ARF and subsequent rheumatic heart disease.

\section{Objective}

This article explores current recommendations for primary prevention of ARF in Australia.

\section{Discussion}

People at increased risk of ARF should be offered empirical antibiotic treatment of Strep A infections to reduce this risk. People at increased ARF risk include young Aboriginal and Torres Strait Islander people in remote Australia as well as those with a personal or family history of ARF and people from migrant communities in urban areas, including Māori and Pacific Island people. Riskstratified primary prevention can reduce the inequitable burden of ARF and rheumatic heart disease in Australia.
ACUTE RHEUMATIC FEVER (ARF) is an abnormal immune reaction, occurring weeks after Streptococcus pyogenes (Strep A) infection in susceptible young people. Susceptibility to ARF is likely to be driven by recurrent Strep A infections in early life, leading to immune priming. ${ }^{1}$ This means that children exposed to Strep A early and more frequently - for example, in crowded living environments or with inadequate access to hygiene infrastructure - are at greatest risk of ARF. Aboriginal and Torres Strait Islander people are disproportionately affected by these factors and account for nearly $90 \%$ of the approximately $500 \mathrm{ARF}$ diagnoses in Australia each year. ${ }^{2}$ The other $10 \%$ of people who develop ARF include non-Indigenous people born in Australia (approximately 7\%) and First Nations peoples from other countries and migrants from low- or lower middleincome countries (approximately $3 \%$ ). ${ }^{2}$ In Australia, the highest burden of ARF is in remote Northern Australia, although confirmed cases occur in all capital cities, and it is important for general practitioners (GPs) to be aware of ARF and the clinical diagnostic features regardless of where they work. ${ }^{2,3}$ Severe or recurrent episodes of ARF lead to the chronic heart valve damage of rheumatic heart disease (RHD), which leads in turn to heart failure and

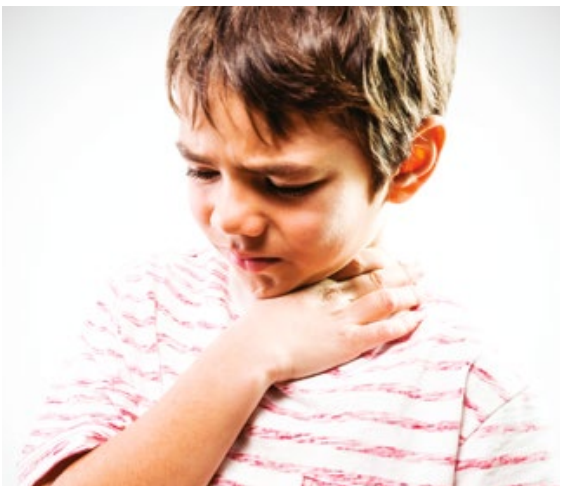

an increased risk of stroke, arrythmia, bacterial endocarditis and pregnancy complications. The disease burden experienced by individuals with RHD is substantial but able to be mitigated with high-quality secondary prevention. ${ }^{4}$ This article focuses on the role of GPs and other health professionals in the primary prevention of ARF.

\section{The role of Strep A infections} in acute rheumatic fever

The risk of Strep A throat infection precipitating ARF has been recognised since the 1950 s. ${ }^{5}$ Without treatment, approximately $3 \%$ of people with streptococcal pharyngitis will go on to develop ARF. ${ }^{6}$ There is also growing evidence that Strep A skin infections (impetigo) can precipitate ARF. This hypothesis emerged in Australia with the observation that skin sores are common among Aboriginal and Torres Strait Islander communities with high rates of ARF, whereas reported throat infections are relatively rare. ${ }^{7}$ Similar epidemiological patterns have been described internationally, and a well-described case report from $\mathrm{New}$ Zealand has provided new evidence that Strep A skin infection can lead to ARF. ${ }^{8,9}$ This is important because skin infections 
are common; half of all children in remote communities have skin infections at any one time, primarily caused by Strep A. ${ }^{10,11}$ Skin problems, including skin sores, are also common presentations in regional and urban general practice. ${ }^{12}$

\section{Primary prevention of acute rheumatic fever}

Primary prevention involves the identification of Strep A infections and delivery of appropriate antibiotic treatment to prevent ARF (as distinct from antibiotic treatment to shorten the duration of infection, heal the sores or provide symptomatic relief). The evidence that treatment of Strep A throat infections can prevent ARF is strong, albeit based on historic studies. ${ }^{13}$ Prompt treatment with oral penicillin is reported to reduce the attack rate of ARF following Strep A throat infection by approximately $70 \%$, increasing to $80 \%$ if a single intramuscular injection of benzathine benzylpenicillin $G$ (BPG) is given. Benefit has been reported within nine days of symptom onset, although lowest risk is likely to be achieved with earlier commencement of effective antibiotics. ${ }^{14,15}$ There is no empirical evidence that antibiotic treatment of Strep A skin infections has the same effect in reducing ARF risk.
However, it is biologically plausible that timely treatment of skin infection would limit the immune response, immune priming and molecular mimicry that underpin the pathophysiology of ARF. Additionally, treatment of skin sores is important to limit spread to other sites or transmission to other people, and reduce direct complications (skin infection including cellulitis, sepsis and bone infections) and indirect complications (glomerulonephritis). Australian public health guidelines require patients to receive an effective antibiotic for 24 hours prior to return to school, to limit onward transmission. ${ }^{16}$

\section{Acute rheumatic fever risk assessment and treatment choices \\ Sore throat}

Approximately $70 \%$ of young people presenting to general practice with a sore throat have a viral infection and should not receive antibiotics. ${ }^{17}$ A number of antimicrobial stewardship studies and programs have contributed to appropriately discouraging antibiotic prescriptions for tonsillitis in Australia. ${ }^{18,19}$ However, these efforts should not preclude antibiotic prescriptions for primary prevention of ARF among young people who are at higher risk of immune-mediated consequences. A diagnosis of ARF or RHD has lifelong medical and social effects. ${ }^{20}$ The devastating consequences of RHD can be prevented by primary prevention for people at greatest risk of ARF.

There are at least seven clinical guidelines for management of sore throat in Australia, with variable guidance on ARF risk stratification. ${ }^{21,22}$ The most recent is The 2020 Australian guideline for prevention, diagnosis and management of acute rheumatic fever and rheumatic heart disease, 3 rd edition. ${ }^{23}$ This guideline recommends a risk stratification approach for ARF to identify those at highest risk of ARF and hence increase the likelihood of prescribing antibiotics to treat Strep A infections to prevent ARF (Table 1).

This approach to ARF risk stratification is the most appropriate driver of antibiotic therapy decisions in the Australian setting. Clinical scoring tools, including the Centor score, have been developed to help assess the likelihood of Strep A sore throat relative to other causes. However, positive and negative predictive values are relatively low, and scores focus on diagnostic probability rather than the risk of autoimmune sequelae from untreated infection. ${ }^{23,24}$

People who may be at high risk of ARF require primary prevention antibiotic treatment for sore throat (Figure 1).

\section{Table 1. Risk groups for primary prevention of ARF ${ }^{23}$}

\begin{tabular}{ll}
\hline At high risk & Living in an ARF-endemic setting* \\
& Aboriginal and/or Torres Strait Islander peoples living in rural or remote settings \\
& Aboriginal and/or Torres Strait Islander peoples, and Māori and/or Pacific Islander peoples living in \\
& metropolitan households affected by crowding and/or lower socioeconomic status \\
& Personal history of ARF/RHD and aged $<40$ years
\end{tabular}

May be at high risk Family or household recent history of ARF/RHD

Household overcrowding ( $>2$ people per bedroom) or low socioeconomic status

Migrant or refugee from low- or middle-income country and their children

Additional considerations Prior residence in a high ARF risk setting

that increase risk Frequent or recent travel to a high ARF risk setting

Aged 5-20 years (the peak years for ARF)

\footnotetext{
*This refers to populations where community ARF/RHD rates are known to be high, eg ARF incidence >30/100,000 per year in 5-14-year-olds or RHD all-age prevalence $>2 / 1000$

$A R F$, acute rheumatic fever; $R H D$, rheumatic heart disease

Reproduced with permission from RHDAustralia (ARF/RHD writing group), The 2020 Australian guideline for prevention, diagnosis and management of acute rheumatic fever and rheumatic heart disease, 3rd edn, Casuarina NT, RHDAustralia, 2020.
} 
First-line treatment is a single immediate dose of intramuscular BPG delivered according to evidence-based guidance to minimise the pain of injection delivery. ${ }^{21,23}$ When intramuscular injection is declined or unavailable, a 10-day course of phenoxymethylpenicillin can be provided. ${ }^{23}$ Although no other antibiotic regimen has shown efficacy in primary prevention risk reduction, clinical guidelines provide second-line options for patients with documented penicillin hypersensitivity or allergy (usually a cephalosporin or macrolide antibiotic)..$^{23}$ Supportive therapy including analgesia should be offered.

\section{Skin sores}

There is also a range of guidelines for treatment of Strep A skin infection, including the National healthy skin guideline: For the prevention, treatment and public health control of impetigo, scabies, crusted scabies and tinea for Indigenous populations and communities in Australia. ${ }^{25}$ Skin infections are generally not caused by viruses, and the decision to initiate antibiotics for skin infection is usually clearer than for sore throat. This decisionmaking process is outlined in Figure 2. The evidence for topical treatment, such as mupirocin ointment for people with few sores, is well established and derives from studies in non-endemic, urban settings. ${ }^{26}$ Systemic treatment with oral cotrimoxazole is more appropriate as primary prevention for people at risk of ARF. ${ }^{27,28}$ A three-day course of twice-daily trimethoprim/ sulfamethoxazole should be offered; where injectable therapy is preferred, a single dose of BPG may be given. ${ }^{25} \mathrm{~A}$ limited number of other oral alternatives are also available. Topical treatment is not recommended in settings with a high burden of skin disease because of rapid development of resistance. ${ }^{25}$ People with skin sores should also be offered examination for scabies infestation, tinea or head lice, which predispose to skin damage and subsequent

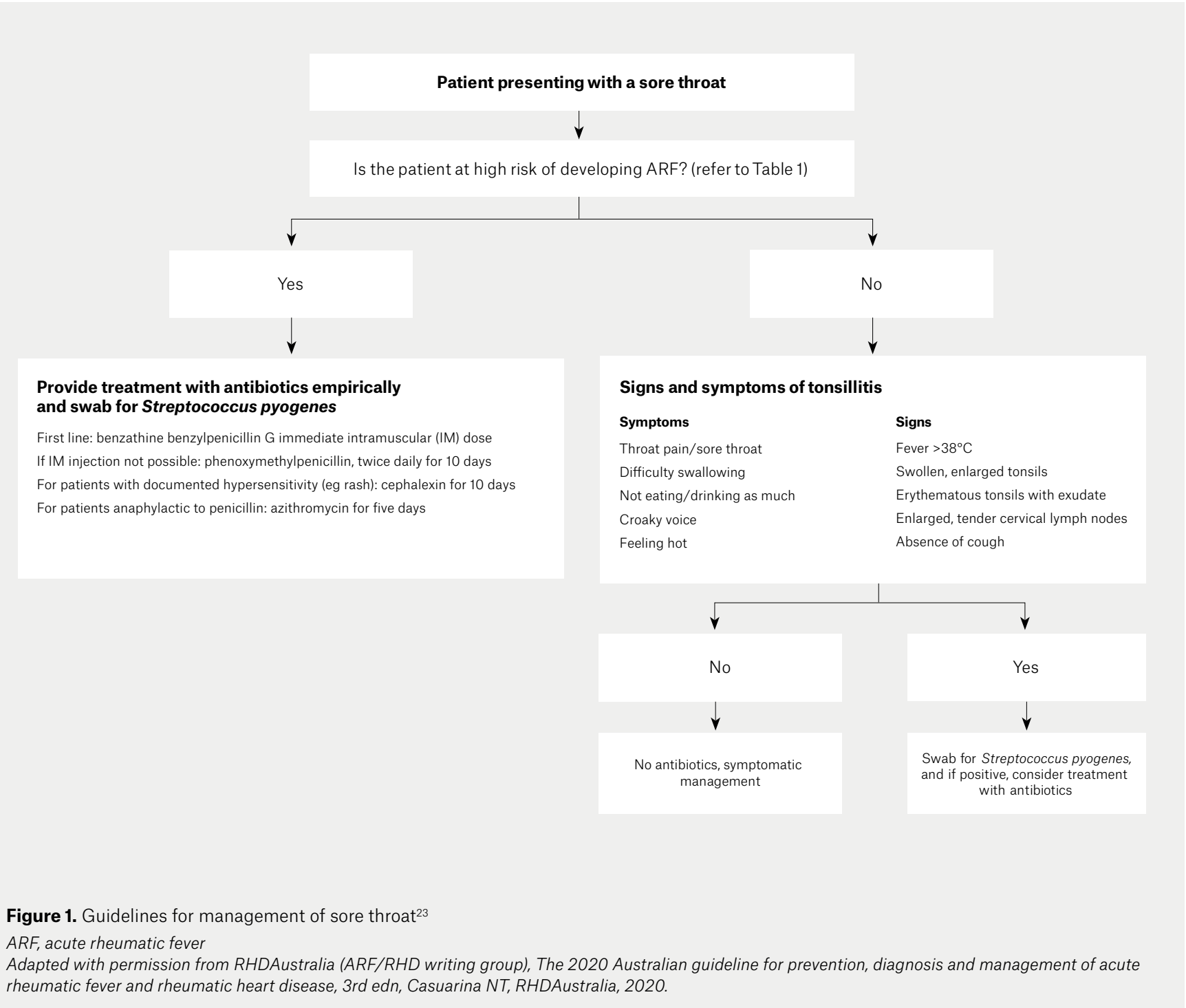


Strep A infection. Sores should be covered with a watertight dressing and antibiotic therapy commenced before returning to school. ${ }^{25}$ Daily handwashing has been shown to reduce incidence of impetigo internationally. ${ }^{29}$ Where services exist, referral to discuss environmental health and housing infrastructure issues may be appropriate to support health hygiene practices.

\section{Microbial testing}

Empirical antibiotic treatment for people at high risk of ARF should be started for sore throat and skin sores without waiting for microbiological confirmation of
Strep A. A throat swab should be taken in the case of sore throat, and oral antibiotics can be ceased if the swab result is negative for Strep A. ${ }^{23}$ Molecular point-of-care testing (PoCT) for Strep A sore throat may support decision making regarding antibiotic use, but emerging PoCT guidance has not yet been incorporated into clinical guidelines. ${ }^{24}$ Wound swabs are generally not indicated for skin sores unless there has been treatment failure. ${ }^{25}$

\section{Conclusion}

There is a persistently high incidence of ARF in Australia driven by inequities in environmental health and access to acceptable healthcare for primary prevention. These inequities mean the burden of ARF is concentrated among - but not exclusive to - Aboriginal and Torres Strait Islander peoples. Development of ARF can be prevented by early detection and appropriate antibiotic treatment of sore throats and skin sores for higher-risk groups. Clinical guidelines that assess treatment needs in the context of population risk are important tools to reduce recognised underlying health disparities. Primary care has a critical role in encouraging, validating and providing best practice care for young people attending with these common and potentially serious infections.

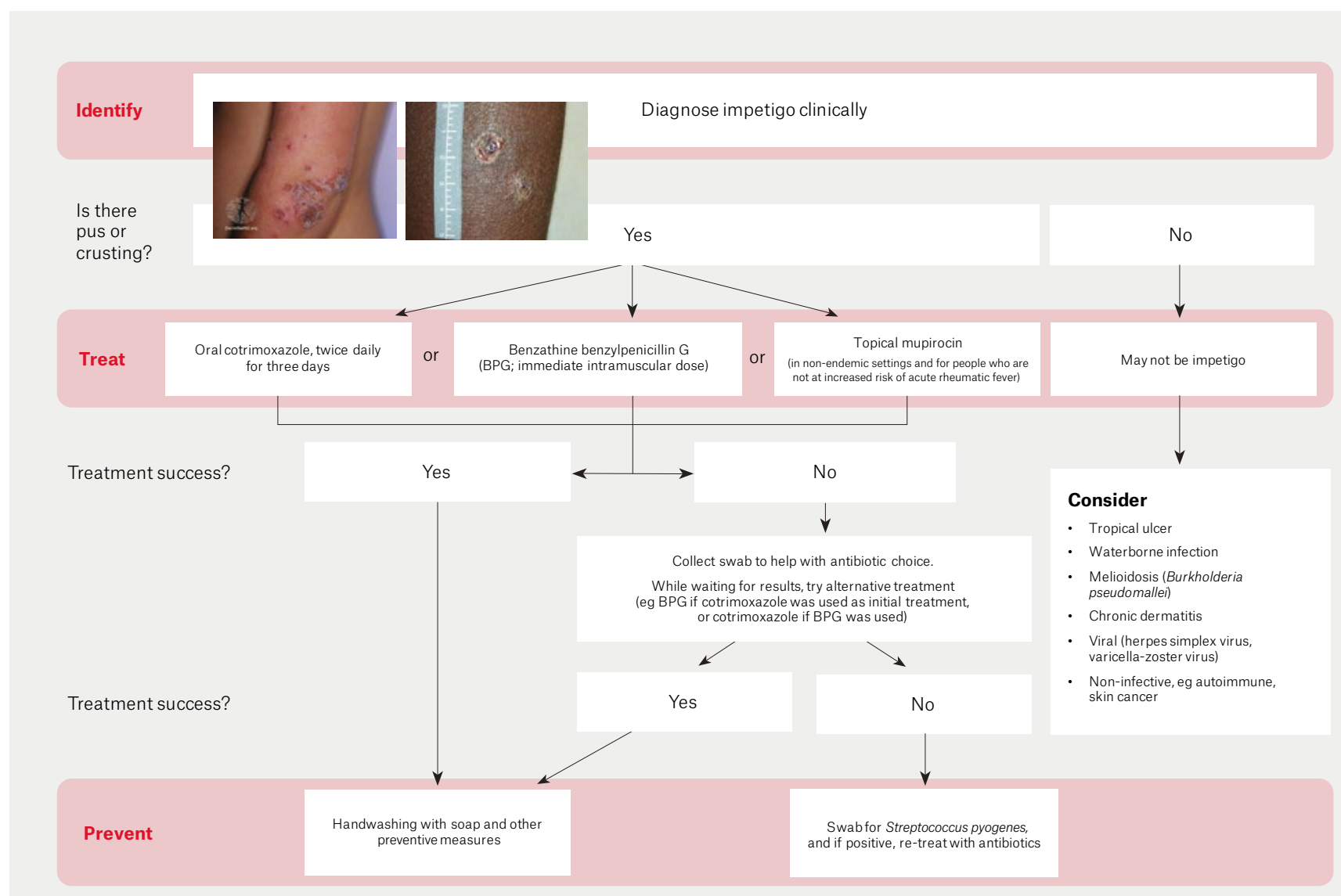

Figure 2. Guidelines for management of skin sores ${ }^{25}$

Adapted with permission from The Australian Healthy Skin Consortium, National healthy skin guideline: For the prevention, treatment and public health control of impetigo, scabies, crusted scabies and tinea for Indigenous populations and communities in Australia, 1st edn, Nedlands, WA: Telehealth Kids Institute, 2018. Clinical images from National healthy skin guideline: For the prevention, treatment and public health control of impetigo, scabies, crusted scabies and tinea for Indigenous populations and communities in Australia, 1st edn and DermNet NZ (Creative Commons Attribution-NonCommercialNoDerivs 3.0 (New Zealand) at https://creativecommons.org/licenses/by-nc-nd/3.0/nz/legalcode). 


\section{Authors}

Rosemary Wyber MBChB, MPH, FRACGP, The George Institute for Global Health, NSW; END RHD Head of Strategy, Telethon Kids Institute, WA; Senior Adjunct Research Fellow, University of Western Australia, WA

Asha C Bowen BA, MBBS, DCH, FRACP, PhD, Head Skin Health Wesfarmers Centre for Vaccines and Infectious Diseases, Telethon Kids Institute, WA; Associate Professor, University of Western Australia, WA; Paediatric Infectious Diseases Specialist, Perth Children's Hospital, WA; Honorary Fellow, Menzies School of Health Research, Charles Darwin University, NT

Anna P Ralph BMedSci, MPH, DTMH, FRACP, PhD, Professor, Head of Global Health Division and Senior Clinical Research Fellow, Menzies School of Health Research, Charles Darwin University, NT; General Medicine and Infectious Diseases Specialist, Royal Darwin Hospital, NT

David Peiris MBBS, MIPH, PhD, FRACGP, FARGP, Director, Global Primary Health Care Program The George Institute for Global Health, NSW; Professor, Faculty of Medicine, The University of New South Wales, NSW; General Practitioner, Glebe Family Medical Practice, NSW

Competing interests: None.

Funding: None.

Provenance and peer review: Commissioned, externally peer reviewed.

Correspondence to:

Rosemary.Wyber@telethonkids.org.au

\section{Acknowledgments}

RW is supported by a National Health and Medical Research Council (NHMRC) Postgraduate Scholarship (1151165). AB is supported by an NHMRC Investigator Award (1175509). APR is supported by an NHMRC Fellowship (1142011). DP is supported by a NHMRC Fellowship (1143904) and a National Heart Foundation of Australia Fellowship (101890).

\section{References}

1. Raynes JM, Frost HR, Williamson DA, et al. Serological evidence of immune priming by group A streptococci in patients with acute rheumatic fever. Front Microbiol 2016;7:1119. doi: 10.3389/ fmicb.2016.01119.

2. Katzenellenbogen JM, Bond-Smith D, Seth RJ, et al. Contemporary incidence and prevalence of rheumatic fever and rheumatic heart disease in Australia using linked data: The case for policy change. J Am Heart Assoc 2020;9(19):e016851. doi: 10.1161/JAHA.120.016851.

3. Oliver J, Osowicki J, Cordell B, Hardy M, Engelman D, Steer AC. Incidence of acute rheumatic fever and rheumatic heart disease in Melbourne, Australia from 1937 to 2013. J Paediat Child Health 2020;56(9):1408-13. doi: 10.1111/ jpc.14950.

4. de Dassel J, Lennon D, Dougherty S, Ralph A Secondary prevention of acute rheumatic fever and rheumatic heart disease. In: Dougherty $S$ Carapetis J, Zühlke L, Wilson N, editors. Acute rheumatic fever and rheumatic heart disease. St Louis, MO: Elsevier, 2020; p. 207-34.

5. Denny FW, Wannamaker LW, Brink WR, Rammelkamp CH Jr, Custer EA. Prevention of rheumatic fever; treatment of preceding streptococci infection. JAMA 1950;143(2):151-53. doi: 10.1001/jama.1950.02910370001001.

6. Carapetis JR, Currie BJ, Mathews JD. Cumulative incidence of rheumatic fever in an endemic region:
A guide to the susceptibility of the population? Epidemiol Infect 2000;124(2):239-44. doi: 10.1017/ s0950268800003514.

7. McDonald M, Currie BJ, Carapetis JR. Acute rheumatic fever: A chink in the chain that links the heart to the throat? Lancet Infect Dis 2004:4(4):240-45. doi: 10.1016/S14733099(04)00975-2.

8. O'Sullivan L, Moreland NN, Webb RH, Upton A, Wilson NJ. Acute rheumatic fever after group A Streptococcus pyoderma and group G Streptococcus pharyngitis. Pediat Infect Dis 2017:36(7):692-94. doi: 10.1097/ INF.0000000000001558.

9. Parks T, Smeesters PR, Steer AC. Streptococcal skin infection and rheumatic heart disease. Curr Opin Infect Dis 2012;25(2):145-53. doi: 10.1097/ QC0.0b013e3283511d27.

10. Davidson L, Knight J, Bowen AC. Skin infections in Australian Aboriginal children: A narrative review. Med J Aust 2020;212(5):231-37. doi: 10.5694/ mja2.50361.

11. Bowen AC, Tong SY, Chatfield MD, Carapetis JR. The microbiology of impetigo in Indigenous children: Associations between Streptococcus pyogenes, Staphylococcus aureus, scabies, and nasal carriage. BMC Infect Dis 2014;14:727. doi: 10.1186/s12879-014-0727-5.

12. Gorges $\mathrm{H}$, Heal $\mathrm{C}$, van Driel $\mathrm{M}$, et al. Prevalence and associations of general practice registrars' management of impetigo: A cross-sectional analysis from the Registrar Clinical Encounters in Training (ReCEnT) study. Dermatol Pract Concept 2020;10(2):e2020043. doi: 10.5826/dpc.1002a43.

13. Ali SKM, Engel ME, Zühlke L, Jack SJ. Primary prevention of acute rheumatic fever and rheumatic heart disease. In: Dougherty S, Carapetis J, Zühlke L, Wilson N, editors. Acute rheumatic fever and rheumatic heart disease. St Louis, MO: Elsevier, 2020; p. 195-206.

14. Robertson KA, Volmink JA, Mayosi BM. Antibiotics for the primary prevention of acute rheumatic fever: A meta-analysis. BMC Cardiovasc Disord 2005;5(1):11. doi: 10.1186/1471-2261-5-11.

15. Spinks A, Glasziou PP, Del Mar CB. Antibiotics for sore throat. Cochrane Database Syst Rev 2013(11):CD000023. doi: 10.1002/14651858. CD000023.pub4.

16. Communicable Diseases Network Australia Acute rheumatic fever (ARF) and rheumatic heart disease (RHD). CDNA national guidelines for public health units. Canberra, ACT: CDNA, 2018.

17. Oliver J, Malliya Wadu E, Pierse N, Moreland NJ, Williamson DA, Baker MG. Group A Streptococcus pharyngitis and pharyngeal carriage: A meta-analysis. PLoS Negl Trop Dis 2018:12(3):e0006335. doi: 10.1371/journal. pntd.0006335.

18. Choosing Wisely Australia. Antibiotic resources for clinicians. Strawberry Hills, NSW: NPS MedicineWise, [date unknown]. Available at www.choosingwisely.org.au/resources/healthprofessionals/antibiotic-resources-for-clinicians [Accessed 25 March 2021].

19. Magin PJ, Morgan S, Tapley A, et al. Reducing general practice trainees' antibiotic prescribing for respiratory tract infections: An evaluation of a combined face-to-face workshop and online educational intervention. Educ Prim Care 2016;27(2):98-105. doi: 10.1080/14739879.2015.1106085.

20. Haynes E, Mitchell A, Enkel S, Wyber R, Bessarab D. Voices behind the statistics: A systematic literature review of the lived experience of rheumatic heart disease. Int
Environ Res Public Health 2020;17(4):1347 doi: 10.3390/ijerph17041347.

21. Tarca AJ, Hand RM, Wyber R. Call for a national sore throat guideline. Med J Aust 2019;210(10):477-477.e1. doi: 10.5694/mja2.50166

22. Expert Group for Rheumatology. Rheumatology: Sore throat. In: eTG complete [Internet]. West Melbourne, Vic: Therapeutic Guidelines Limited, 2021.

23. RHDAustralia (ARF/RHD writing group). The 2020 Australian guideline for prevention, diagnosis and management of acute rheumatic fever and rheumatic heart disease. 3rd edn. Casuarina, NT: RHDAustralia, 2020.

24. Gunnarsson RK, Orda U, Elliott B, et al. Improving antibiotics targeting using PCR point of care testing. Aust J Gen Pract 2021:50(1-2):76-83. doi: 10.31128/AJGP-07-20-5518.

25. The Australian Healthy Skin Consortium. National healthy skin guideline: For the prevention, treatment and public health control of impetigo, scabies, crusted scabies and tinea for Indigenous populations and communities in Australia. 1st edn. Nedlands, WA: Telehealth Kids Institute, 2018.

26. Koning S, van der Sande R, Verhagen AP, et al. Interventions for impetigo. Cochrane Database Syst Rev 2012;1(1):CD003261. doi: 10.1002/14651858.CD003261.pub3.

27. Expert Group for Antibiotic. Antibiotic: Impetigo. In: eTG complete [Internet]. West Melbourne, Vic: Therapeutic Guidelines Limited, 2021.

28. Bowen AC, Tong SY, Andrews RM, et al. Shortcourse oral co-trimoxazole versus intramuscular benzathine benzylpenicillin for impetigo in a highly endemic region: An open-label, randomised, controlled, non-inferiority trial. Lancet 2014;384(9960):2132-40. doi: 10.1016/ S0140-6736(14)60841-2.

29. Luby SP, Agboatwalla M, Feikin DR, et al. Effect of handwashing on child health: A randomised controlled trial. Lancet 2005;366(9481):225-33. doi: 10.1016/S0140-6736(05)66912-7.

correspondence ajgp@racgp.org.au 